\title{
Cerebral infarction caused by endocarditis in a patient with COVID-19
}

Doo Hyuk Kwon, MD'; Jae-Seok Park, MD²; Hyung Jong Park, MD'; Sung-II Sohn, MD'; Jeong-Ho Hong, MD, PhD ${ }^{1}$

'Department of Neurology, Keimyung University School of Medicine, Daegu, Republic of Korea ${ }^{2}$ Department of Internal Medicine, Keimyung University School of Medicine, Daegu, Republic of Korea

\section{Journal of Neurocritical Care \\ CASE REPORT \\ Received: August 6, 2020 \\ Revised: August 19, 2020 \\ Accepted: August 20, 2020 \\ Corresponding Author: Jeong-Ho Hong, MD, PhD \\ Department of Neurology, Keimyung University School of Medicine, 1035 Dalgubeol-daero, Dalseo-gu, Daegu 42601, Korea \\ Tel: +82-53-258-7839 \\ Fax: +82-53-258-4380 \\ E-mail: neurohong79@gmail.com}

\begin{abstract}
Background: Coronavirus disease 2019 (COVID-19) has become a worldwide health threat due to its highly contagious nature and severe complications. The authors experienced a case of cerebral infarction caused by endocarditis in a patient infected with severe acute respiratory syndrome coronavirus 2 (SARS-CoV-2).

Case Report: A 65-year-old male patient with COVID-19 was admitted to our hospital for cohort isolation. On day 11 after admission, the patient suddenly presented right-sided hemiparesis. Computed tomography of the brain showed multiple hypodense lesions, which confirmed acute cerebral infarction. Transthoracic echocardiogram revealed a vegetation of about $5 \mathrm{~mm}$ on the mitral valve and endocarditis was diagnosed as the cause of cerebral infarction.

Conclusion: Cerebral infarction is one of the complications of COVID-19 and can occur at any time during the course of the disease. Hence, a neurologist has an important role in the medical team involved in COVID-19 treatment.
\end{abstract}

Keywords: COVID-19; Heart injury; Endocarditis; Cerebral infarction; Neurologist

\section{INTRODUCTION}

Coronavirus disease 2019 (COVID-19) is a newly identified infectious disease with rapid human to human transmission capacity. COVID-19 can result in several fatal complications such as acute respiratory distress syndrome, RNAaemia, acute cardiac injury, acute kidney injury, secondary infection, and septic shock $[1,2]$. Since February 21, 2020, the authors of this paper have been working at a hospital designated for treating COVID-19 patients and by June 15, 2020, 1,027 COVID-19 patients had been hospitalized. Our critical care team implemented a collaborative approach for intensive care unit patients with severe acute respiratory syndrome coronavirus 2 (SARS-CoV-2) infection enabling simultaneous co-management of pulmonological, neurocritical, and nutritional services. Here, we report a case of multiple cerebral infarctions in a patient hospitalized with COVID-19.

\section{CASE REPORT}

A 65-year-old South Korean male patient with COVID-19 was

(C) 2020 The Korean Neurocritical Care Society

This is an Open Access article distributed under the terms of the Creative Commons Attribution Non-Commercial License (http://creativecommons.org/licenses/by-nc/4.0/) which permits unrestricted noncommercial use, distribution, and reproduction in any medium, provided the original work is properly cited. 
admitted to our hospital for cohort isolation. The patient had no underlying conditions and developed cough and sputum 5 days prior to diagnosis. On admission, the patient's vital signs were recorded as a temperature of $38.0^{\circ} \mathrm{C}$, blood pressure of $135 / 88 \mathrm{~mm} \mathrm{Hg}$, heart rate of 78 beats per minute, respiratory rate of 22 breaths per minute, and oxygen saturation of $95 \%$ on room air. Chest computed tomography (CT) showed pneumonia in the lower lobes of both lungs. We started treatment with lopinavir/ritonavir and hydroxychloroquine, which are known to be effective against COVID-19 $[1,2]$. Additionally, ceftriaxone and azithromycin were simultaneously administered as empirical antibiotics to treat pneumonia.

Six days later, pneumonia gradually worsened, and the patient was put on mechanical ventilation in the intensive care unit as his blood pressure had dropped $(90 / 60 \mathrm{~mm} \mathrm{Hg})$, heart and respiratory rate had increased ( 120 beats per minute and 30 breaths per minute, respectively), and oxygen saturation levels were below $85 \%$. Based on the results of blood culture (positive for Pseudomonas aeruginosa) and drug sensitivity test, antibiotic treatment was changed to include piperacillin/tazobactam and levofloxacin. Inotropes were administered to raise the patient's blood pressure, and $0.4-0.6 \mu \mathrm{g} / \mathrm{kg} /$ $\mathrm{hr}$ of dexmedetomidine and $0.1-0.2 \mu \mathrm{g} / \mathrm{kg} / \mathrm{min}$ of remifentanil were additionally administered during mechanical ventilation.
On day 11 after admission, when the patient's vital signs stabilized, we gradually reduced sedative administration, and the patient began to recover consciousness. However, the patient suddenly developed right-sided hemiparesis (Medical Research Council grade 3). He had a National Institute of Health Stroke Scale score of 7 (partial hemianopia, 1; facial palsy, 1; right arm motor drift, 2; right leg motor drift, 2; and dysarthria, 1). Emergency brain CT with intracranial CT angiography was performed. The brain CT showed multiple hypodense lesions with surrounding edema in different areas of the brain (Fig. 1A and B), which confirmed acute cerebral infarction. Since the intracranial CT angiography was normal, cardioembolic stroke was suspected and transthoracic echocardiogram (TTE) was performed to assess the cause of the stroke. TTE showed a vegetation of approximately 5 $\mathrm{mm}$ on the mitral valve (Fig. 1C and D) and endocarditis was diagnosed as the cause of cerebral infarction. Aspirin $(100 \mathrm{mg})$ that was administered at the time of diagnosis of cerebral infarction was discontinued on suspicion of infectious endocarditis. Antimicrobial therapy with piperacillin/tazobactam and levofloxacin was administered based on blood culture (positive for P. aeruginosa) and antibiotic sensitivity test. Two weeks later, the patient was released from quarantine after two consecutive negative findings on
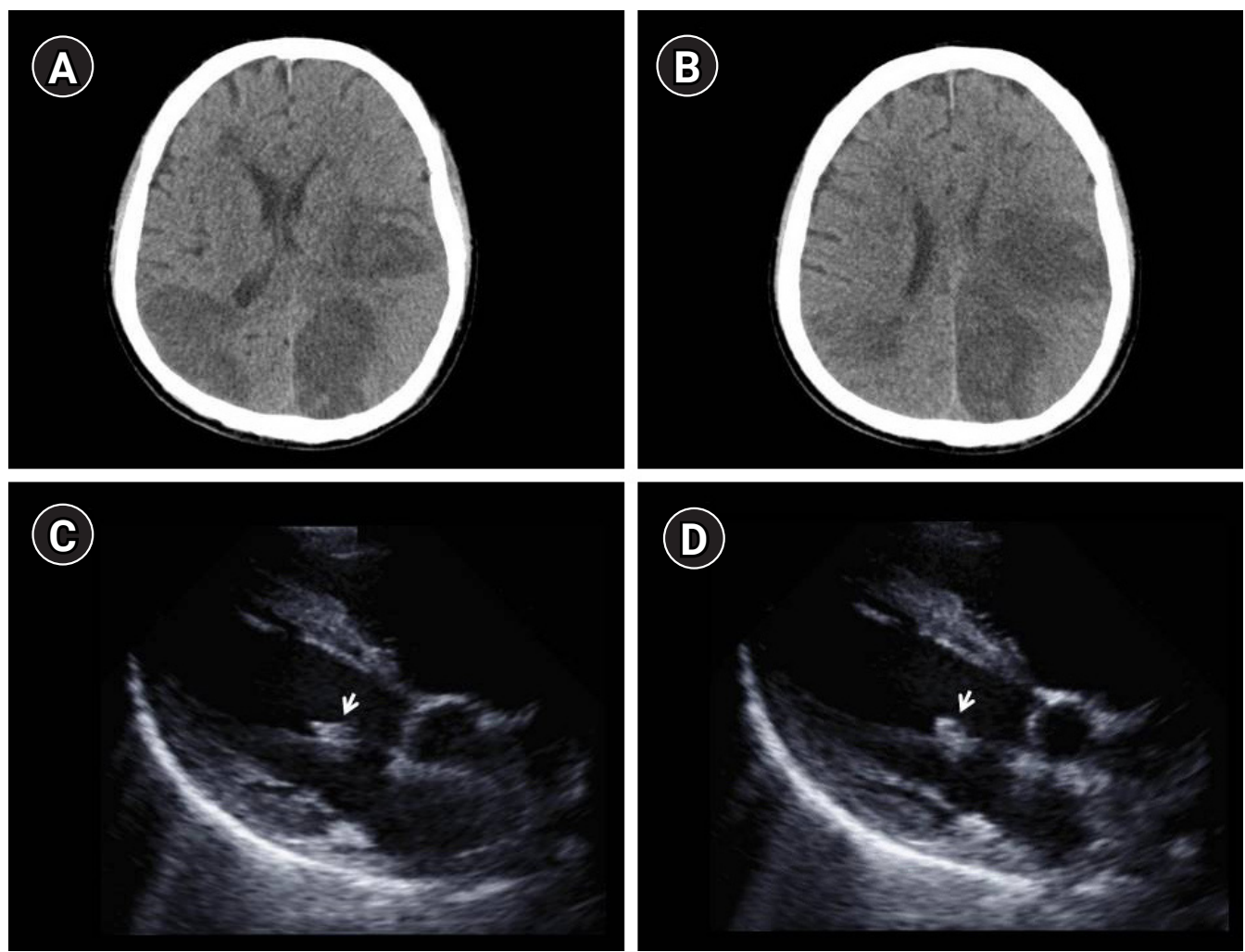

Fig. 1. Findings of brain computed tomography (CT) and transthoracic echocardiogram (TE). (A, B) Brain $C T$ demonstrates multiple hypodense lesions with surrounding edema in different areas of the brain. (C, D) In the TTE (supine view), a mobile and irregularly shaped echodense mass of approximately $1.0 \times 0.5 \mathrm{~cm}$ is attached to the anterior mitral valve (white arrows). 
COVID-19 real-time reverse transcription polymerase chain reaction testing and was transferred to another tertiary hospital (not a COVID-19 designated hospital) for further treatment.

\section{DISCUSSION}

Recently published studies report that COVID-19 could be accompanied by acute cardiac injury (defined as blood levels of hypersensitive troponin I above the 99th percentile upper reference limit $(>28 \mathrm{pg} / \mathrm{mL}$ ) or new abnormalities shown on electrocardiography and echocardiography), secondary infection, and sepsis $[1,2]$. The vegetation of endocarditis is initiated through bacteremia, which then binds and attaches to the damaged endothelium. Thromboplastin, which is secreted by tissue factors in the damaged endothelium, causes platelet aggregation and cleavage of fibrinogen to fibrin. Acute cardiac injury, secondary infections, and sepsis can cause endocarditis $[3,4]$. In our study, P. aeruginosa was reported in blood culture after the patient was confirmed to have COVID-19. Hence, endocarditis can occur due to complications of COVID-19 accompanied by secondary bacterial infection. Despite improved management, endocarditis remains associated with high mortality and serious complications [3]. Symptomatic neurological complications appear in $15 \%-30 \%$ of endocarditis patients and occur primarily due to embolism from vegetations [4]. Neurologic symptoms most occur often before or during endocarditis diagnosis, but new or recurrent events may occur after endocarditis diagnosis. Clinical presentation is variable and may include multiple symptoms or signs in the same patient. Typically, focal signs are diagnosed due to ischemic stroke. Further, transient ischemic attack, intracerebral or subarachnoid hemorrhage, brain abscess, meningitis, and toxic encephalopathy can be present, and there is evidence that $35 \%-60 \%$ of patients with endocarditis develop additional silent cerebral embolisms [5].

We believe that this is the first reported case of cerebral infarction caused by endocarditis in a patient with COVID-19. Several complications can result from COVID-19 and acute cerebral infarction can further potentially occur during the course of this disease $[1,2,6]$. Due to the lack of equipment required for neurological disease assessment and inaccessibility of medical staff in our designated hospital optimized for COVID-19 treatment, it was difficult to perform diagnosis of stroke by physical examination and evaluation. However, it is clear that neurologists have an important role besides respiratory and infectious physicians in the treatment of COVID-19.

\section{ARTICLE INFORMATION}

\section{Ethics statement}

This case was reviewed and approved by the Institutional Review Board of Keimyung University Dongsan Hospital (IRB No. 202009-054). Informed consent was waived by the Board.

\section{Conflict of interest}

Dr. JH Hong is an editorial board member of the journal but was not involved in the peer reviewer selection, evaluation, or decision process of this article. There are no other potential conflicts of interest relevant to this article to declare.

\section{Funding}

This work was supported by the Research Program of Medicity Daegu Council funded by Daegu Metropolitan City (No. COVID19 DM08).

\section{ORCID}

Doo Hyuk Kwon https://orcid.org/0000-0001-7541-9226

Jae-Seok Park

Hyung Jong Park

https://orcid.org/0000-0002-5751-7209

Sung-Il Sohn

https://orcid.org/0000-0002-6112-2939

Jeong-Ho Hong

https://orcid.org/0000-0002-6900-1242

https://orcid.org/0000-0002-8235-9855

\section{Author contributions}

Conceptualization: DHK, JHH. Data curation: DHK, JSP, HJP, JHH. Formal analysis: all authors. Funding acquisition: DHK. Investigation: HJP, SIS. Supervision: JSP, HJP, SIS. Visualization: DHK, HJP, JHH. Writing-original draft: DHK, JHH. Writingreview \& editing: DHK, JHH.

\section{REFERENCES}

1. Huang C, Wang Y, Li X, Ren L, Zhao J, Hu Y, et al. Clinical features of patients infected with 2019 novel coronavirus in Wuhan, China. Lancet 2020;395:497-506.

2. Chen N, Zhou M, Dong X, Qu J, Gong F, Han Y, et al. Epidemiological and clinical characteristics of 99 cases of 2019 novel coronavirus pneumonia in Wuhan, China: a descriptive study. Lancet 2020;395:507-13.

3. Thuny F, Grisoli D, Collart F, Habib G, Raoult D. Management of infective endocarditis: challenges and perspectives. Lancet 2012;379:965-75.

4. Murdoch DR, Corey GR, Hoen B, Miró JM, Fowler VG Jr, Bayer AS, et al. Clinical presentation, etiology, and outcome of infective endocarditis in the 21st century: the International Col- 
laboration on Endocarditis-Prospective Cohort Study. Arch Intern Med 2009; 169:463-73.

5. Snygg-Martin U, Gustafsson L, Rosengren L, Alsiö A, Ackerholm P, Andersson R, et al. Cerebrovascular complications in patients with left-sided infective endocarditis are common: a prospective study using magnetic resonance imaging and neu- rochemical brain damage markers. Clin Infect Dis 2008;47:2330.

6. Mao L, Jin H, Wang M, Hu Y, Chen S, He Q, et al. Neurologic manifestations of hospitalized patients with coronavirus disease 2019 in Wuhan, China. JAMA Neurol 2020;77:1-9. 